\title{
The Prognostic Value of Residual Nonrectal Inflammation in Ulcerative Colitis
}

\author{
Eun Ae Kang \\ Department of Internal Medicine and Institute of Gastroenterology, Yonsei University College of Medicine, Seoul, Korea
}

\section{Article Info}

Received October 23, 2021

Revised November 2, 2021

Accepted November 3, 2021

Published online January 7, 2022

\section{Corresponding Author}

Eun Ae Kang

ORCID https://orcid.org/0000-0003-0220-937X

E-mail cheerea@gmail.com

\section{To the Editor:}

A recently published retrospective study by Shin et al. ${ }^{1}$ evaluated the prognosis of ulcerative colitis (UC) patients in clinical remission (CR) according to the distribution and severity of endoscopically detected residual inflammation. Patients who achieved endoscopic remission or residual nonrectal inflammation with patch distribution had better clinical outcomes (progression-free survival, progression-free survival) than patients with residual rectal inflammation. Multivariate analysis identified only rectal inflammation as being associated with a higher risk of poor outcomes. This study was the first to evaluate residual inflammation patterns in UC patients in CR and compare the effects of residual inflammation patterns on progression-free survival.

Endoscopic remission, also known as mucosal healing, is considered a therapeutic goal for UC. ${ }^{2}$ However, CR based on patient-reported outcomes is inconsistent with endoscopic findings. A previzous study reported that only half of these patients with CR based on patient-reported outcomes had endoscopic remission. ${ }^{3}$ There have been several studies on the effects of endoscopic residual inflammation in UC. Previous studies have reported that irritable bowel syndrome-like symptoms are associated with residual colonic inflammation in UC patients in $\mathrm{CR}^{4,5}$ One study reported that residual short-segment distal inflammation is not a risk factor for major relapses, provided that endoscopic remission is achieved in the proximal colon. ${ }^{6}$

I would like to raise a few questions to the authors of this study. First, the severity of nonrectal inflammation and rectal inflammation was significantly different $(p<0.001)$ between the groups and there was a wide variation in Ulcerative Colitis Segmental Endoscopic Index (UCSEI) scores. A worse prognosis may be attributable to the severity of endoscopic condition, rather than the distribution of residual rectal inflammation. According to the results presented in Table 2, UCSEI was a significant predictor of poor outcomes in the univariate analysis but not in the multivariate analysis; this result is likely due to the small sample size of this study.

Second, it may be beneficial to discuss the number of patients using topical 5-aminosalicylic acid (5-ASA) suppositories in the nonrectal and rectal inflammation groups since residual rectal inflammation may be affected by the use of topical 5-ASA formulations. Poor adherence and intolerance to 5-ASA are associated with a risk of adverse clinical outcomes. ${ }^{7.8}$ According to the data presented in Supplementary Table 1, there was no between-group difference in the use of medications, but the use of thiopurine or biologics was higher in the rectal inflammation group than in the endoscopic remission or nonrectal inflammation group. It is possible that the rectal inflammation group maintained CR status because the patients used highly effective medications such as immunomodulators or biologics. ${ }^{9,10}$ Therefore, the possibility of bias cannot be ruled out. 
Third, Supplementary Fig. 1 shows the changes in the distribution of residual inflammation according to the distribution of inflammation in UC patients in CR. However, the figure fails to show if clinical outcomes differed according to the change of distribution: from rectal inflammation $(n=51)$ to endoscopic remission $(n=16)$, nonrectal inflammation $(n=2)$, or sustained rectal inflammation $(n=33)$.

In conclusion, as the authors noted, well-designed prospective studies are needed to support the findings of this study. With stronger evidence, customized treatment according to a patient's residual rectal inflammation status will be possible.

\section{CONFLICTS OF INTEREST}

No potential conflict of interest relevant to this article was reported.

\section{ORCID}

Eun Ae Kang https://orcid.org/0000-0003-0220-937X

\section{REFERENCES}

1. Shin J, Kong SM, Kim TJ, et al. Clinical significance of residual nonrectal inflammation in ulcerative colitis patients in clinical remission. Gut Liver 2021;15:401-409.

2. Hibi T, Naganuma M, Oda E, et al. Predictive factors for achievement of mucosal healing by budesonide 2-mg foam in ulcerative colitis: a pooled analysis of data from two clini- cal trials. Intest Res 2020;18:56-68.

3. Dulai PS, Singh S, Jairath V, et al. Prevalence of endoscopic improvement and remission according to patient-reported outcomes in ulcerative colitis. Aliment Pharmacol Ther 2020;51:435-445.

4. Ishihara S, Kawashima K, Fukuba N, et al. irritable bowel syndrome-like symptoms in ulcerative colitis patients in clinical remission: association with residual colonic inflammation. Digestion 2019;99:46-51.

5. Fukuba N, Ishihara S, Tada Y, et al. Prevalence of irritable bowel syndrome-like symptoms in ulcerative colitis patients with clinical and endoscopic evidence of remission: prospective multicenter study. Scand J Gastroenterol 2014;49:674680.

6. Asonuma K, Kobayashi T, Nakano M, et al. Residual shortsegment distal inflammation has no significant impact on the major relapse of extensive ulcerative colitis. Inflamm Bowel Dis 2022;28:200-207.

7. Mizuno S, Ono K, Mikami Y, et al. 5-Aminosalicylic acid intolerance is associated with a risk of adverse clinical outcomes and dysbiosis in patients with ulcerative colitis. Intest Res 2020;18:69-78.

8. Sood A, Ahuja V, Midha V, et al. Colitis and Crohn's Foundation (India) consensus statements on use of 5-aminosalicylic acid in inflammatory bowel disease. Intest Res 2020;18:355-378.

9. Hibi T, Kamae I, Pinton P, et al. Efficacy of biologic therapies for biologic-naïve Japanese patients with moderately to severely active ulcerative colitis: a network meta-analysis. Intest Res 2021;19:53-61.

10. Ooi CJ, Hilmi I, Banerjee R, et al. Best practices on immunomodulators and biologic agents for ulcerative colitis and Crohn's disease in Asia. Intest Res 2019;17:285-310. 